\title{
Desorption of hydrolyzed poly(AM/DMDAAC) from bentonite and its decomposition in saltwater under high temperatures
}

https://doi.org/10.1515/epoly-2019-0056

Received July 05, 2019; accepted August 03, 2019.

\begin{abstract}
Under harsh conditions, the desorption of polyampholytes from bentonite (Bent) can affect the performance of drilling fluids. To study the desorption of polyampholyte from bentonite, partially hydrolyzed copolymers of acrylamide and diallyl dimethyl ammonium chloride (HPAD), containing carboxyl groups, quaternary ammonium groups and amide groups was synthesized via free radical copolymerization followed by hydrolyzation. The molecular structure of HPAD was characterized by ${ }^{1} \mathrm{H}$ NMR and ${ }^{13} \mathrm{C}$ NMR. The adsorption equilibrium of HPAD on Bent in the presence of $10 \mathrm{wt} \% \mathrm{NaCl}$ was $106 \mathrm{mg} \cdot \mathrm{g}^{-1}$. The adsorptiondesorption behavior of HPAD on Bent was studied using a high pressure and high temperature (HPHT) filtration apparatus, to obtain the filtrate liquid and filter cakes. The content of HPAD in the filtration and the filter cakes was determined via UV and element analysis, respectively. As the temperature increased, the desorption of HPAD from Bent accelerated owing to molecular thermal motion and thermal degradation of the adsorptive groups. Notably, the decomposition rate of the amide group was more than twice that of the quaternary ammonium group. The critical temperature for HPAD desorption was $135^{\circ} \mathrm{C}$, as the decomposition of the adsorptive groups became predominant over intensified molecular thermal motion at high temperatures.
\end{abstract}

\footnotetext{
* Corresponding author: Ling Lin, School of Chemistry and Chemical Engineering, Southwest Petroleum University, Chengdu, Sichuan Province, 610500, China, Oil \& Gas Field Applied Chemistry Key Laboratory of Sichuan Province (Southwest Petroleum University), Chengdu, Sichuan Province, 610500, email: cowbolinling@aliyun. com; Tel.: +86-28-83037306

Chenliang Shi and Yifan Mao, School of Chemistry and Chemical Engineering, Southwest Petroleum University, Chengdu, Sichuan Province, 610500, China; email: chenliang.shi@aliyun.com (Chenliang Shi), 924849515@qq.com (Yifan Mao)

Xin Li, School of Petroleum Engineering, Southwest Petroleum University, Chengdu, Sichuan Province, 610500, email:up96157@gmail.com
}

Keywords: bentonite; acrylamide; diallyl dimethyl ammonium chloride; desorption; decomposition

$\begin{array}{ll}\text { Abbreviations } & \\ \text { Bent } & \text { bentonite } \\ C_{\text {amide group }} & \text { content of amide groups } \\ C_{H P A D} & \begin{array}{l}\text { content of HPAD } \\ \text { hydrolyzed poly(acrylamide/diallyl } \\ \text { HPAD }\end{array} \\ \text { dimethyl ammonium chloride } \\ \text { HPHT } & \text { high-pressure and high-temperature } \\ n_{\mathrm{C}} & \text { molar percentage of carbon } \\ n_{\mathrm{N}} & \text { molar percentage of nitrogen } \\ \text { PAD } & \text { poly(acrylamide/diallyl dimethyl } \\ & \text { ammonium chloride) } \\ w_{\mathrm{C}} & \text { weight percentage of carbon } \\ w_{\mathrm{N}} & \text { weight percentage of nitrogen }\end{array}$

\section{Introduction}

Polyampholytes have broad applications as chemical additives, e.g. filtration reducers, in water-based drilling fluids $(1,2)$. In mud, both polyampholytes and bentonite (Bent) play as the main components contributing to the viscosity of drilling fluids. Polyampholytes ionize in aqueous solution and adsorb on Bent via both electrostatic attraction and hydrogen bonding (3). This process provides Bent with both electrostatic repulsion and entropic repulsion, which contributes to the colloidal stability of Bent. However, if the amphoteric polymers rapidly desorb from Bent under harsh conditions, the colloidal properties of Bent suffered considerably, resulting in the deterioration of rheological properties of drilling fluids. Therefore, the desorption of polyampholyte from Bent is a crucial factor in the performance of drilling fluid under harsh conditions, especially under high temperature, high pressure and high salinity $(3,4)$. 
Previous research on the adsorption of amphoteric polymers in aqueous solution has mainly focused on the adsorption amount and the conformation of polymer chains, typically at moderate temperatures and in deionized water $(5,6)$. The adsorption kinetics of polyampholyte on expanded perlite under $25^{\circ} \mathrm{C}$ to $55^{\circ} \mathrm{C}$ were described by the pseudo first-order kinetics model (7). The adsorption of polyampholytes or polyacrylamidebased materials on Bent has been studied previously $(3,8)$. Yan and Zhang reported that the adsorption of charged PAMs on montmorillonite and kaolinite followed the order cationic $>$ non-ionic $>$ anionic, and that the desorption of PAMs from clay seemed to be impossible (9).

To the best of our knowledge, the adsorption/ desorption of polymers on adsorbents, especially Bent, in aqueous solution at high temperatures has rarely been studied. Wang et al. found that the adsorption of polyvinyl alcohol on Bent increased from $30^{\circ} \mathrm{C}$ to $60^{\circ} \mathrm{C}$ (10). This phenomenon may be a result of accelerated adsorption compared with an original low adsorption or the creation of new adsorptive sites on the adsorbent surface (11). Other papers reporting the effect of temperature on the adsorption behavior of polyampholyte have mainly focused on mild temperatures $(12,13)$. The degradation of polyacrylamide-based polymers under high temperature has been studied by Jiang etal. (14). The authors argued that carboxylic groups of partially hydrolyzed polyacrylamide, which were the hydrolyzation products of amide groups, acted as catalysts of the further hydrolyzation of amide groups under heating.

As the desorption mechanism of polyampholyte has rarely been studied under harsh conditions, this study was aimed at obtaining a quantitative description of the factors that affect the desorption of partially hydrolyzed poly(acrylamide/diallyl dimethyl ammonium chloride) (HPAD) at high temperatures in saltwater. Both molecular thermal motion and adsorptive groups degradation were expected to influence the desorption of HPAD from Bent. The former factor was found to have the greatest influence on HPAD desorption from Bent at temperatures below $135^{\circ} \mathrm{C}$, whereas the latter factor became more influential at higher temperatures.

\section{Materials and methods}

\subsection{Materials and preparation}

Acrylamide, $\mathrm{NaHSO}_{3}, \mathrm{MnCl}_{2}, \mathrm{NaOH}$, methyl orange, indigo carmine, $\mathrm{AgNO}_{3}, \mathrm{~K}_{2} \mathrm{CrO}_{4}, \mathrm{Na}_{2} \mathrm{CO}_{3}, \mathrm{NaCl}$ and ethanol were purchased from Chengdu Kelong Chemical Company, China. Diallyl dimethyl ammonium chloride (60 wt $\%$ in water) was obtained from Sinopharm Chemical Reagent Company, Shanghai, China. All of these chemicals were analytic reagent and used as received.

Bent was provided by Zhongfei Xiazijie Bentonite Company, Xinjiang, China. Bent was purified according to the following procedure: first, $5 \mathrm{wt} \%$ Bent and $0.25 \mathrm{wt} \%$ $\mathrm{Na}_{2} \mathrm{CO}_{3}$ were added to deionized water, and the mixture was well mixed and hydrated at room temperature for $24 \mathrm{~h}$; second, the mixture was filtered using vacuum pump ( $15-20 \mu \mathrm{m}$ pore size filter paper); third, the filtration was centrifugated at $10000 \mathrm{rpm}$ for $20 \mathrm{~min}$, and Bent at the bottom of centrifuge tube was dried at $70^{\circ} \mathrm{C}$ for $16 \mathrm{~h}$ under vacuum $\left(10^{-3}\right.$ Torr); finally, dried Bent was ground and sifted with a screen mesh (\#100) and then stored in a desiccator until use. The components of Bent are provided in the supporting files, as determined using China's O\&G Industrial Standard SY/T 5163-2010.

\subsection{Polymer synthesis}

Poly(acrylamide/diallyl dimethyl ammonium chloride) (PAD), was synthesized as follows: first, acrylamide and diallyl dimethyl ammonium chloride were mixed together in deionized water at a molar ratio of 5:1; second, the mixture was purged with $\mathrm{N}_{2}$ for 20 min and the flask was sealed with a rubber plug; third, $0.1 \mathrm{wt} \% \mathrm{MnCl}_{2}$ in deionized water and $0.05 \% \mathrm{NaHSO}_{3}$ in deionized water were injected into the mixture, respectively, and the flask was heated in water bath at $60^{\circ} \mathrm{C}$ for $12 \mathrm{~h}$; fourth, the reaction product was precipitated (twice) from water using $200 \mathrm{~mL}$ of $\mathrm{CH}_{3} \mathrm{CH}_{2} \mathrm{OH}$, which changed the polarity of the solution and therefore lowered the solubility of the polymers, and then dried under vacuum over under $60^{\circ} \mathrm{C}$ for $12 \mathrm{~h}$.

HPAD was prepared by hydrolyzing PAD in alkaline solution $(10 \mathrm{wt} \% \mathrm{NaOH})$ under $85^{\circ} \mathrm{C}$ for $16 \mathrm{~h}$. Subsequently, the product was precipitated (four times) from water using $200 \mathrm{~mL}$ of $\mathrm{CH}_{3} \mathrm{CH}_{2} \mathrm{OH}$, and then dried under vacuum $\left(10^{-3}\right.$ Torr) at $60^{\circ} \mathrm{C}$ for $12 \mathrm{~h}$.

\subsection{Sample characterization}

The cation exchange capacity of the Bent $(75.81 \mathrm{cmol} / 100 \mathrm{~g})$ was determined according to China industrial standard SY/T 5395-2016. The content of quaternary ammonium units and carboxyl units in polymers was determined using a titration method in accordance with China Standard GB 
12005.6-89. The cationic group content was measured by titrating a $0.05 \mathrm{~mol} \cdot \mathrm{L}^{-1} \mathrm{AgNO}_{3}$ solution against an aqueous PAD solution, with $\mathrm{K}_{2} \mathrm{CrO}_{4}$ ( $5 \mathrm{wt} \%$ in water) as the indicator. The titration endpoint was characterized by a change of the solution color from bright yellow to brick red. HPAD was assumed to inherit the cationic group content of PAD. The anionic group content was measured by titrating a $0.1 \mathrm{~mol} \cdot \mathrm{L}^{-1} \mathrm{HCl}$ solution against an aqueous HPAD solution, with a mixture of $0.1 \mathrm{wt} \%$ methyl orange and $0.25 \mathrm{wt} \%$ indigo carmine as the indicator, and the endpoint was characterized by a color change from yellowish green to French gray. The intrinsic viscosities of polymers were tested in a $1 \mathrm{~mol} \cdot \mathrm{L}^{-1} \mathrm{NaCl}$ aqueous solution using an Ubbelohde capillary viscometer with a capillary diameter of 0.55-0.60 mm (Shanghai Shenyi Glass Instrument Co. Ltd., China) at $30 \pm 0.1^{\circ} \mathrm{C}$, according to China Standard $\mathrm{GB} / \mathrm{T}$ 1632-1993. The infrared transmission spectra $\left(500 \mathrm{~cm}^{-1}\right.$ to $4000 \mathrm{~cm}^{-1}, 0.25 \mathrm{~cm}^{-1}$ resolution) of the polymers were obtained with WQF-520 FT-IR (Beijing Rayleigh Analytical Instrument Corporation, China). The ${ }^{1} \mathrm{H}$ NMR and ${ }^{13} \mathrm{C}$ NMR spectra (narrow bore, static, relaxation time $=$ $2 \mathrm{~s}$ ) of the polymers in $\mathrm{D}_{2} \mathrm{O}$ were recorded using a Bruker Ascend $^{\mathrm{TM}} 400 \mathrm{MHz}$ NMR spectrometer at $25^{\circ} \mathrm{C}$. UV-Vis absorption spectra (190-1100 nm, $2 \mathrm{~nm}$ bandwith) were collected using an LS6 spectrophotometer (Shanghai INESA Scientific Instrument Co., Ltd, China) at $30^{\circ} \mathrm{C}$. After treatment using the "starch - cadmium iodide" method, the amide groups of HPAD exhibited an absorption maximum at $525 \mathrm{~nm}$ (15). The linear relationship between the amide group content and the absorbance was used to calculate the concentration of HPAD in aqueous solution. The analysis of elements and function groups on the surface of adsorbed Bent were determined using X-ray photoelectron spectroscopy (XPS, Thermo Scientific Escalab 250Xi) with a Mg Ka X-ray source (1254 eV of photons). The high-resolution scans were performed over the 392-410 eV ranges (N1s spectra) for polymer samples. The software Avantage (Thermo Scientific, America) was used to analyze the obtained XPS spectra peaks.

The adsorption of polymers on Bent under $30^{\circ} \mathrm{C}$ was tested according to the following procedure: first, a Bent solution and a polymer solution were prepared and aged separately at room temperature for $12 \mathrm{~h}$; second, the two solutions were mixed, and $10 \mathrm{wt} \% \mathrm{NaCl}$ was added, and the mixture was aged at $30^{\circ} \mathrm{C}$ in a water bath; third, the mixture was centrifuged at $10000 \mathrm{rpm}$ for $20 \mathrm{~min}$; fourth, the supernatant was treated using the "starch-cadmium iodide" method, and then the polymer content was determined via UV-Vis spectroscopy, as described above; fifth, the precipitated Bent was rinsed with deionized water for three times, and then dried under vacuum $\left(10^{-3} \mathrm{Torr}\right)$ at $70^{\circ} \mathrm{C}$ for $16 \mathrm{~h}$. The weight percentage of carbon $\left(w_{\mathrm{C}}\right)$ and nitrogen $\left(w_{\mathrm{N}}\right)$ in the Bent samples $(15 \pm 2 \mathrm{mg})$ was tested in a combustion tube on a Vario EL III elemental analysis device (Elementar, Germany) in CHNS mode at $1150^{\circ} \mathrm{C}$. The molar ratio of $\mathrm{C} / \mathrm{N}$ can be calculated as below:

$$
\frac{n_{C}}{n_{N}}=\frac{w_{C} / 12}{w_{N} / 14}
$$

The polymer desorption experiments under high temperatures $\left(\geq 100^{\circ} \mathrm{C}\right)$ were carried out on a high pressure and high temperature (HPHT) filtrate apparatus (Qingdao Tongchun Company, China) as shown in Figure 1. first, a saltwater mixture containing both Bent and the polymer was prepared following the same steps under $30^{\circ} \mathrm{C}$; second, the suspension was transferred to a steel oven with a filter paper (pore size of $1 \mu \mathrm{m}$ ) at the bottom, and the oven was heated to target temperatures in the HPHT filtrate apparatus; third, the bottom valve of the oven was opened every $15 \mathrm{~min}$ to collect the filtration, and a filter cake was obtained when all the liquid in the oven was released; fourth, the filter cake was rinsed with deionized water three times at room temperature; fifth, the polymer contents in the filtration and filter cakes were determined according to the adsorption test procedure at $30^{\circ} \mathrm{C}$.
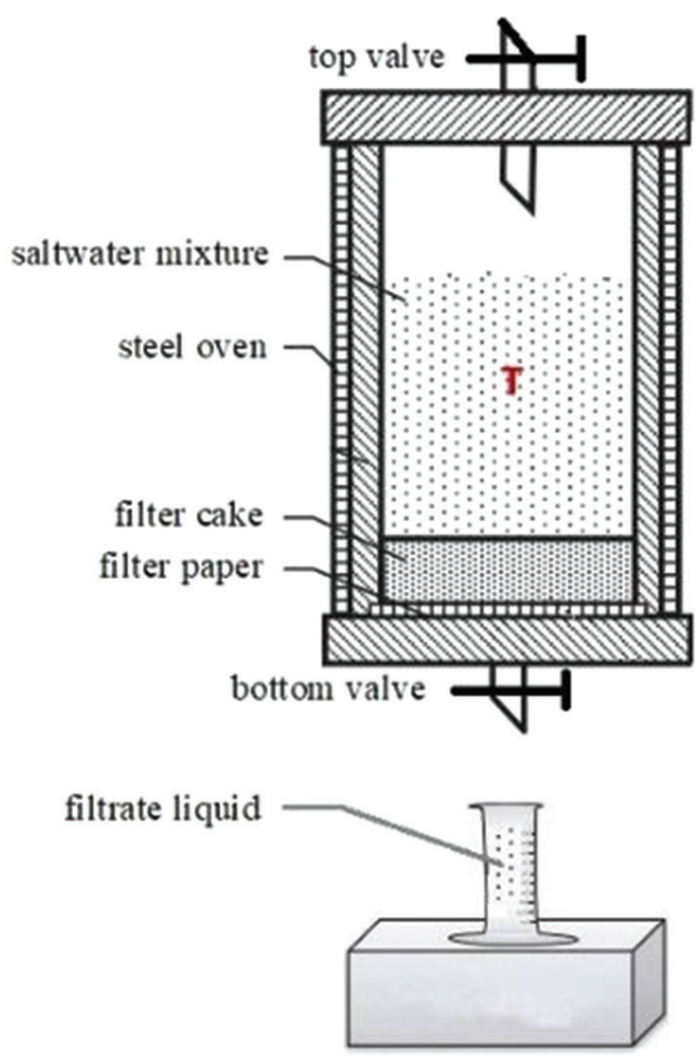

Figure 1: Desorption experiments using HPHT filtrate apparatus. 
Table 1: Viscosities and compositions of polymer samples.

\begin{tabular}{lcccc}
\hline Sample & $\begin{array}{c}{[\eta]} \\
\left(\mathrm{mL} \cdot \mathrm{g}^{-1}\right)\end{array}$ & $\begin{array}{c}\text { Carboxyl unit } \\
(\mathrm{mol})\end{array}$ & $\begin{array}{c}\text { Quaternary } \\
\text { ammonium } \\
\text { unit (mol\%) }\end{array}$ & $\begin{array}{c}\text { Amide unit } \\
(\mathrm{mol} \%)\end{array}$ \\
\hline PAD & 432.3 & 0 & 10.48 & 89.52 \\
HPAD & 606.8 & 22.09 & 10.48 & 67.43 \\
\hline
\end{tabular}

\section{Results}

\subsection{Molecular structure characterization}

The intrinsic viscosity and composition of polymer samples, as determined by viscometry and titration methods, are listed in Table 1.

The amphoteric polymer HPAD was the hydrolyzed product of PAD, and the content of cationic groups was assumed to be equal in these two polymers. The polyampholyte HPAD interacted with Bent via electrostatic attraction and hydrogen bonding, with both quaternary ammonium groups and amide groups acting as the adsorptive groups which interacted with the surfaces and edges of Bent in aqueous solutions.

The synthesis of HPAD was confirmed by the NMR test results as shown in Figure 2.

Signals corresponding to amide groups, carboxyl groups, and quaternary ammonium groups were observed in both the ${ }^{1} \mathrm{H}$ NMR and ${ }^{13} \mathrm{C}$ NMR spectra of HPAD. In addition, although both monomers (acrylamide and diallyl dimethyl ammonium chloride) contained $\mathrm{C}=\mathrm{C}$ groups, no $\mathrm{C}=\mathrm{C}$ signals (normally located at $100-150 \mathrm{ppm}$ ) were observed in the ${ }^{13} \mathrm{C}$ NMR spectrum of HPAD. These result indicated that HPAD was successfully prepared via free radical polymerization followed by hydrolyzation.

\subsection{Adsorption of HPAD on Bent under $30^{\circ} \mathrm{C}$}

The effect of stirring time and the amount of polymer added on the adsorption of HPAD on Bent at $30^{\circ} \mathrm{C}$ were studied, as shown in Figure 3.

In the adsorption kinetics experiment, the original mixture contained $0.1 \mathrm{wt} \%$ HPAD, $1 \mathrm{wt} \%$ Bent, and $10 \mathrm{wt} \% \mathrm{NaCl}$. The $\mathrm{pH}$ of the mixture was approximately 7 . As shown in Figure 3a, adsorption equilibrium was reached after $75 \mathrm{~min}$. Therefore, a mixing time of $75 \mathrm{~min}$ was used to prepare saltwater mixture for the following adsorption experiments.

Next, the relationship between the concentration of HPAD and its adsorption on Bent at $30^{\circ} \mathrm{C}$ was determined
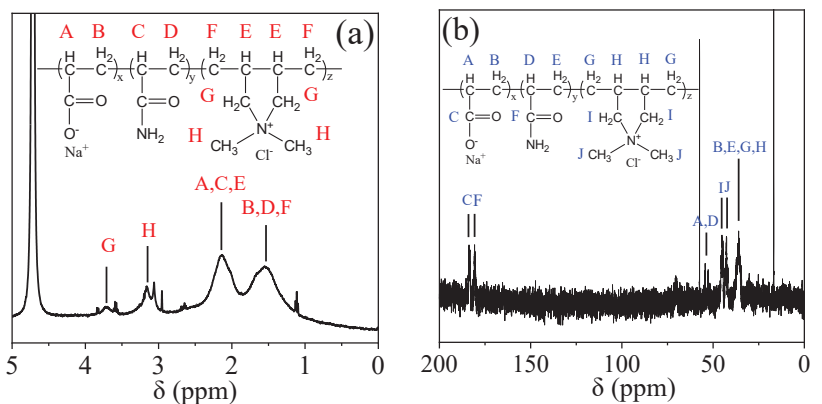

Figure 2: ${ }^{1} \mathrm{H}$ NMR spectrum (a) and ${ }^{13} \mathrm{C}$ NMR spectrum (b) of HPAD.
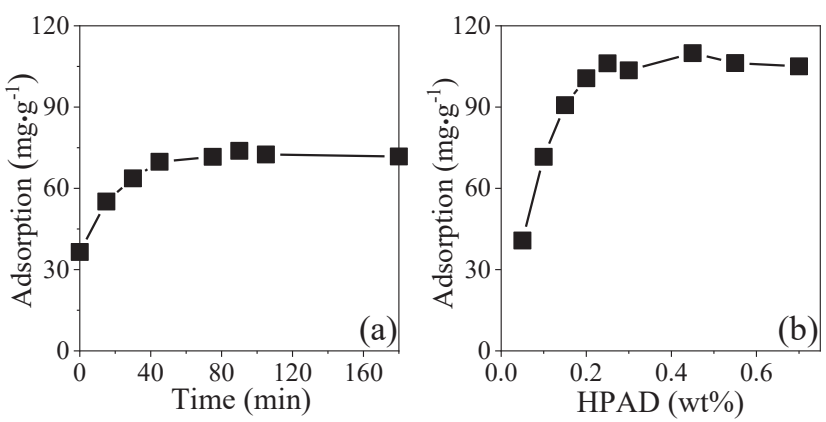

Figure 3: The adsorption kinetics (a) and thermodynamics (b) of HPAD on Bent at $30^{\circ} \mathrm{C}$.

(Figure 2b) by adding various amount of HPAD to $1 \mathrm{wt} \%$ Bent suspension with $10 \mathrm{wt} \% \mathrm{NaCl}$. Adsorption equilibrium was observed when the concentration of HPAD reached $0.3 \mathrm{wt} \%$, and the maximum adsorption amount was approximately $106 \mathrm{mg} \cdot \mathrm{g}^{-1}$.

\subsection{Desorption of HPAD from Bent at high temperatures}

Subsequently, the desorption of HPAD from Bent in the presence of $\mathrm{NaCl}$ at high temperatures was studied using an HPHT filtrate apparatus. Before being heated, $0.1 \mathrm{wt} \%$ HPAD was allowed to reach adsorption equilibrium on Bent in saltwater mud (1 wt $\%$ Bent $+10 \mathrm{wt} \% \mathrm{NaCl})$ at $30^{\circ} \mathrm{C}$ by stirring the mixture for $75 \mathrm{~min}$. After reaching the target temperature, both the filtration and the filter cake were collected to analyze the HPAD contents. The former was assumed to contain HPAD desorbed from Bent, whereas the later was assumed to contain HPAD still adsorbed on Bent; a proportion of decomposed HPAD, in the form of small molecules, could not be detected in either the filtration or the filter cake.

As HPAD is applied as a chemical additive of waterbased drilling fluids in the oil and gas industry, it will be subjected to high temperatures, normally ranging 
from $110^{\circ} \mathrm{C}$ to $200^{\circ} \mathrm{C}$, during drilling engineering (1). Therefore, the temperature of $115-175^{\circ} \mathrm{C}$ was used in the following desorption experiments. The content of $\operatorname{HPAD}\left(C_{H P A D}\right)$ in the collected filtration was calculated based on the content of amide groups $\left(C_{\text {amide group }}\right)$ in the filtration, as determined using UV-Vis spectroscopy, and the molar percentage of amide group in HPAD (Table 1), as follows:

$$
C_{H P A D}=\frac{C_{\text {amide group }}}{0.6743}
$$

The content of HPAD in in the filtration at various high temperatures is shown in Figure 4.

The content of HPAD in the filtration first increased and then decreased as the temperature increased, indicating that the total amount of adsorbed HPAD and decomposed HPAD first decreased and then increased. This phenomenon was caused by intensified thermal molecular motion and accelerated decomposition of the adsorptive groups. The former factor contributed to the desorption of HPAD from Bent into the filtration while the latter resulted in the detection of less HPAD in either the filtration or filter cakes. As the temperature rose from $115^{\circ} \mathrm{C}$ to $135^{\circ} \mathrm{C}$, desorption resulting from the disruption of the electrostatic interactions between HPAD and Bent was the predominant behavior and had a much greater effect than the decomposition of the adsorptive groups, resulting in a positive correlation between the HPAD content in the filtration and the temperature. As the temperature increased further to above $135^{\circ} \mathrm{C}$, the HPAD content in the filtration decreased because the decomposition of adsorptive groups became more prevalent, leading to a rapid reduction of the polymer content.

The content of HPAD in filter cakes after being heated under $175^{\circ} \mathrm{C}$ was shown in Figure $4 \mathrm{~b}$. The contents
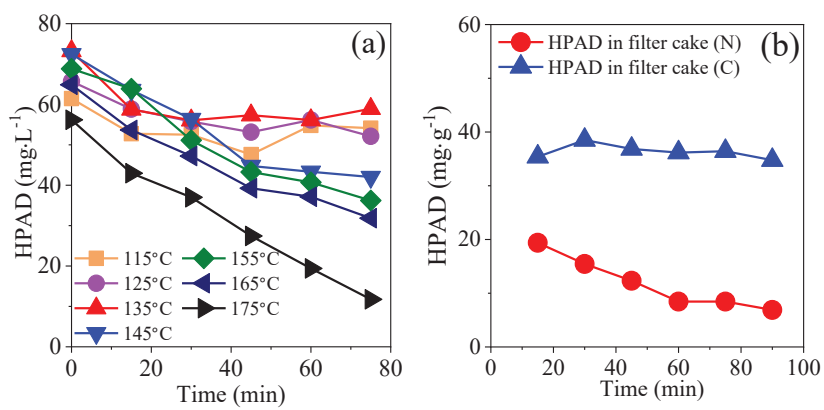

Figure 4: (a) Content of HPAD in the filtration at high temperatures; (b) Amounts of HPAD adsorbed on Bent at $175^{\circ} \mathrm{C}$ ( $\mathrm{N}$ and $\mathrm{C}$ refers to the HPAD content calculated based on $w_{N}$ and $w_{C}$ of the element tests, respectively, as determined by elemental analysis). of HPAD in the filter cakes were calculated based on the content of carbon and nitrogen in the filter cakes $\left(w_{\mathrm{N}}\right.$ and $w_{\mathrm{C}}$, as determined by elemental analysis) and the weight percentage of carbon and nitrogen in HPAD (49.4 wt $\%$ and $12.7 \mathrm{wt} \%$, respectively; Table 2), as follows:

$$
\begin{aligned}
& H P A D_{\text {based on } C}\left(\mathrm{mg} \cdot \mathrm{g}^{-1}\right)=\frac{w_{C} / 0.494}{1-w_{C} / 0.494} \times 100 \\
& H P A D_{\text {based on } N}\left(\mathrm{mg} \cdot \mathrm{g}^{-1}\right)=\frac{w_{N} / 0.127}{1-w_{N} / 0.127} \times 100
\end{aligned}
$$

Interestingly, the content of HPAD in the filter cakes was almost constant if calculated based on $w_{C}$, indicating that the carbon-containing groups in HPAD were not readily decomposed by heating. However, if calculated based on $w_{N}$, the content of HPAD in the filter cakes showed a continuous loss, revealing that the N-based groups were much less stable than alkyl groups. A possible explanation for the difference in behavior is that even the N-based adsorptive groups were decomposed, HPAD chains were still entrapped by adjacent Bent particles and could not be released into the filtration. Furthermore, we can infer that some HPAD decomposed during the original heating process, resulting in the HPAD content in filter cakes calculated based on $w_{\mathrm{N}}$ less than that calculated based on $w_{C}$ at 15 min.

\subsection{Degradation of HPAD at high temperatures}

The degradation of HPAD at high temperatures was examined using $2 \mathrm{wt} \% \mathrm{HPAD}$ in saltwater (10 $\mathrm{wt} \% \mathrm{NaCl}$ ). After stirring for $10 \mathrm{~h}$ under $30^{\circ} \mathrm{C}$, the mixture was aged at varied temperatures for various time. Subsequently, the polymer was precipitated by adding ethanol and then was dried under vacuum $\left(10^{-3}\right.$ Torr $)$ at $60^{\circ} \mathrm{C}$ for $12 \mathrm{~h}$. Finally, the intrinsic viscosities of the aged HPAD samples were determined. Considering that $135^{\circ} \mathrm{C}$ was the critical temperature at which the decomposition of HPAD

Table 2: Effect of aging on relative contents of chemical groups in HPAD.

\begin{tabular}{lc}
\hline Time $(\mathrm{min})$ & $\mathrm{A \delta}_{3.1} / \mathrm{Ad}_{3.5}$ \\
\hline 15 & 1.375 \\
30 & 1.048 \\
45 & 0.786 \\
90 & 0.365 \\
\hline
\end{tabular}

$\delta_{3.1}$ and $\delta_{3.5}$ correspond to $H$ and $G$ (Figure 2a) 


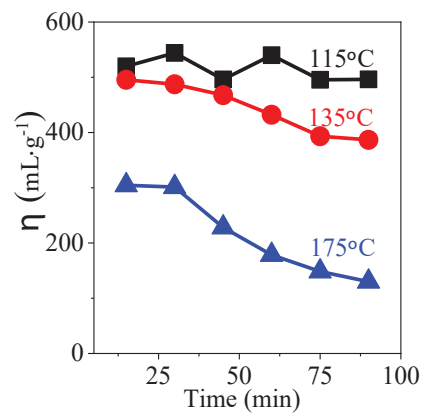

Figure 5: Intrinsic viscosities of HPAD aged under high temperatures.

accelerated, the aging temperatures of $115^{\circ} \mathrm{C}, 135^{\circ} \mathrm{C}$, and $175^{\circ} \mathrm{C}$ were investigated (Figure 5).

Over $90 \mathrm{~min}$, the intrinsic viscosity of HPAD varied little at $115^{\circ} \mathrm{C}$, but it exhibited a decrease at higher temperatures, with almost three-quarters of the initial intrinsic viscosity lost at $175^{\circ} \mathrm{C}$. Pronounced decreases in the intrinsic viscosity were observed at $135^{\circ} \mathrm{C}$ and $175^{\circ} \mathrm{C}$, indicating that the decomposition of HPAD in saltwater was accelerated at the temperatures higher than $115^{\circ} \mathrm{C}$.

To better understand the degradation of the chemical groups along the chains, IR and ${ }^{1} \mathrm{H}$ NMR analysis of the aged samples were conducted. In the IR spectra (Figure 6a), the band near $2930 \mathrm{~cm}^{-1}$ was attributed to the asymmetric stretching vibration of methyl, methylene and methylidyne groups; the band at $1610 \mathrm{~cm}^{-1}$ was attributed to the $\mathrm{C}=\mathrm{O}$ of amide groups (16); the bands at $1450 \mathrm{~cm}^{-1}$ and $1405 \mathrm{~cm}^{-1}$ were attributed to asymmetric bending vibrations and symmetric bending vibrations of methyl, methylene and methylidyne groups; the band near $1310 \mathrm{~cm}^{-1}$ was attributed to $\mathrm{C}-\mathrm{N}$ of five-membered rings. As the treatment temperature increased, the degradation of methyl groups in the five-membered ring became more prominent than the decomposition of other chemical groups.

As shown by the NMR spectra in Figure 6b, the relative contents of chemical groups varied with aging duration. Based on the relative peak areas of $\mathrm{G}$ and $\mathrm{H}$, HPAD suffered a higher loss of $-\mathrm{CH}_{3}$ linked to five-membered ring than $-\mathrm{CH}_{2}-$ moieties in five-membered rings (Table 2), which is in good agreement with the IR analysis results.

\section{Discussion}

The desorption of HPAD from Bent can be attributed to two factors: first, molecular thermal movements of the polyampholyte disrupt the electrostatic attraction
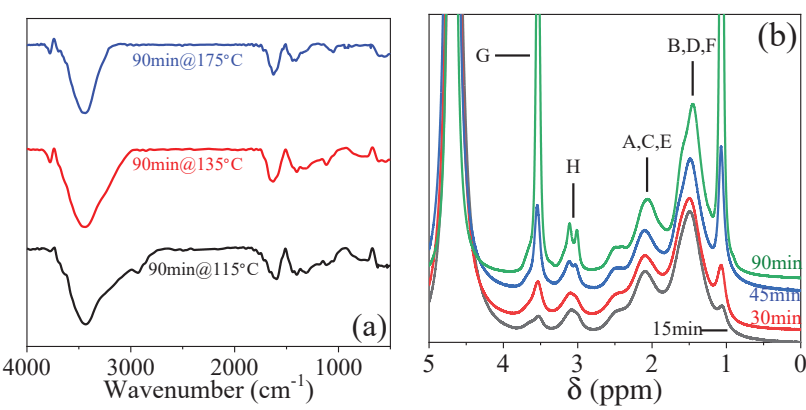

Figure 6: IR (a) and ${ }^{1} \mathrm{H}$ NMR (b) spectra of HPAD aged under different temperatures (the A-H labels are assigned in Figure $2 \mathrm{a}$ ).

between the adsorptive chemical groups and the Bent surface; second, the adsorptive groups, including positively charged quaternary ammonium groups and amide groups, are degraded. Assuming that these factors are the cause of desorption, HPAD in the filtration corresponds to the polymers no longer bound to Bent, as calculated based on the content of amide group with an initial HPAD concentration of $0.1 \mathrm{wt} \%$; HPAD in the filter cakes represents the polyampholyte that still adsorbed on Bent, as calculated based on the content of nitrogen with an initial Bent concentration of $1 \mathrm{wt} \%$; and the remainder corresponds to decomposed HPAD that cannot be detected either in the filtrate liquid or on Bent using titration or element analysis methods. To evaluate the contribution of the two factors to the desorption of HPAD from Bent quantitatively, we calculate the percentage of these three kinds of HPAD as below:

$$
\begin{aligned}
H P A D_{\text {desorbed }}(\%) & =\frac{H P A D_{\text {desorbed }}\left(\mathrm{mg} \cdot \mathrm{L}^{-1}\right)}{H P A D_{\text {original }}\left(\mathrm{mg} \cdot \mathrm{L}^{-1}\right)} \\
& =\frac{C_{\text {amide group }}\left(\mathrm{mg} \cdot \mathrm{L}^{-1}\right)}{0.6743 \times 1000 \mathrm{mg} \cdot \mathrm{L}^{-1}}
\end{aligned}
$$

$$
\begin{aligned}
& H P A D_{\text {adsorbed }}(\%)=\text { Sample }_{\text {original }}(\mathrm{g}) \times \frac{w_{N} / 0.127}{1-w_{N} / 0.127} \\
& \times \frac{\text { Bent }_{\text {original }}(\mathrm{g})}{\text { Sample }_{\text {original }}(\mathrm{g})} \times \frac{1}{H P A D_{\text {original }}(\mathrm{g})} \\
& =\frac{w_{N}}{0.127} \times \frac{1}{1} \times \frac{1}{0.1}=\frac{10 \times w_{N}}{0.127} \\
& H P A D_{\text {decomposed }}(\%)=1-H P A D_{\text {desorbed }}(\%) \\
& -H P A D_{\text {adsorbed }}(\%)
\end{aligned}
$$


The calculated amounts of desorbed HPAD in the filtrate, adsorbed HPAD in the filter cakes, and decomposed HPAD are summarized in Figure 7.

When the saltwater mixture containing HPAD and Bent was heated under $175^{\circ} \mathrm{C}$, Bent-adsorbed HPAD experienced an accelerated desorption and decomposition, as the amount of HPAD adsorbed on Bent was much lower than the amount of desorbed HPAD in the filtration. The difference between the amounts of these two kinds of HPAD became smaller over time, indicating that the content of desorbed HPAD in the filtration declined more rapidly at such a high temperature. However, a steady increase in the calculated percentage of decomposed HPAD was observed. At approximately $30 \mathrm{~min}$, the amounts of decomposed HPAD and desorbed HPAD were approximately equal. Therefore, we can infer that before $30 \mathrm{~min}$, the intensified thermal motion of HPAD dominated the desorption process, whereas the accelerated degradation of adsorptive groups became more important at longer times.

It should be noted that the percentages of desorbed HPAD in the filtration and adsorbed HPAD in the filter cakes (Figure 7) were calculated based on the content of amide groups and the content of $\mathrm{N}$ respectively. However, if the content of adsorbed HPAD in the filter cakes was calculated based on the $\mathrm{C}$ content, the trend should be quite different, according to the data in Figure 4a. We still use the content of $\mathrm{N}$ to calibrate the content of HPAD in the filtration and filter cakes as both types of adsorptive groups in HPAD contain $\mathrm{N}$.

We calculated the $n_{\mathrm{C}} / n_{\mathrm{N}}$ of HPAD in filter cakes under $175^{\circ} \mathrm{C}$ based on $w_{C}$ and $w_{\mathrm{N}}$ values determined by element analysis, as shown in Figure 8a.

As the heating time increased, the $n_{\mathrm{C}} / n_{\mathrm{N}}$ ratio increased from the initial value of 4.52. Acceleration was observed after $60 \mathrm{~min}$, indicating that N-based groups, namely quaternary ammonium groups and amide groups, underwent enhanced decomposition at $175^{\circ} \mathrm{C}$. The $n_{\mathrm{C}} / n_{\mathrm{N}}$ ratio of aged HPAD samples revealed that the loss of $\mathrm{N}$-based groups HPAD in saltwater at $175^{\circ} \mathrm{C}$ was considerably greater than the loss of alkyl groups. Considering that these two types of N-based groups acted as the adsorptive groups, HPAD lost its capacity to adsorb on Bent.

These result was in good agreement with the recognized fact that alkyl groups are much more stable than amide groups and quaternary ammonium groups under high temperatures. The degradation of $-\mathrm{CH}_{3}$ linked to the five-member rings likely accounted for the decrease in the C content, in accordance with the ${ }^{1} \mathrm{H}$ NMR spectrum in Figure 6b. Combined with the drop in the HPAD content in the filtration, as calculated based on amide groups,

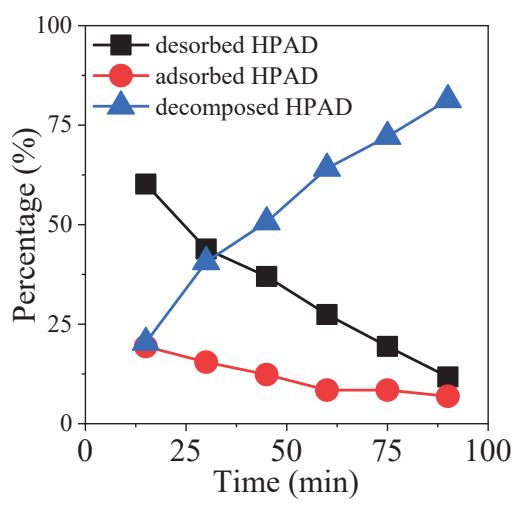

Figure 7: Calculated amounts of desorbed HPAD, adsorbed HPAD and decomposed $\mathrm{HPAD}$ at $175^{\circ} \mathrm{C}$.
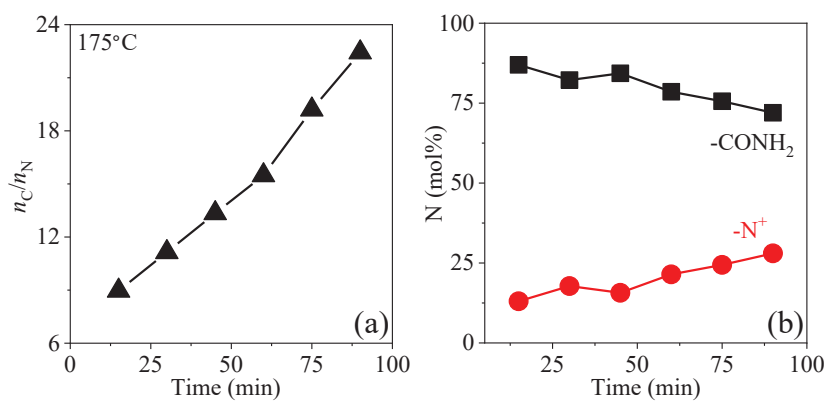

Figure 8: (a) The $\mathrm{C} / \mathrm{N}$ (in molar ratio) of HPAD in filter cakes under $175^{\circ} \mathrm{C}$; (b) N-based groups in $\mathrm{HPAD}$ aged at $175^{\circ} \mathrm{C}$, as determined using XPS.

the huge loss (50\%) of $\mathrm{N}$ content likely corresponds to the decomposition of amide groups rather than quaternary ammonium groups.

To obtain a better understanding of the degradation of $\mathrm{N}$-contained groups, a series of XPS measurements were carried out and the result are summarized in Figure 8b.

The $\mathrm{N}$ content of HPAD in the filtration originated from either quaternary ammonium groups or amide groups. Based on the amide groups in XPS spectra (14), the amount of amide groups decreased continually with increasing heating times, whereas the content of quaternary ammonium groups steadily increased. At $15 \mathrm{~min},-\mathrm{N}^{+}$in the five-member ring accounted for approximately $13 \mathrm{~mol} \%$, corresponding well with the data in Table 1 , while $-\mathrm{N}^{+}$accounted for more than $25 \mathrm{~mol} \%$ at $90 \mathrm{~min}$. Thus, amide groups decomposed much faster than the positively charged groups, indicating that the latter is more likely to act as the adsorptive groups under heating. In aqueous solution, the degradation of amide groups could experience a self-acceleration process, as the $\mathrm{pKa}$ of $\mathrm{H}_{2} \mathrm{O}$ increased at higher temperatures $(17,18)$. 


\section{Conclusion}

In this study, a polyampholyte, HPAD, comprising quaternary ammonium groups, carboxyl groups and amide groups was synthesized via free radical copolymerization followed by hydrolyzation using $\mathrm{NaOH}$. The thermal stability and the adsorption of HPAD on Bent in the saltwater were studied. The maximum adsorption of HPAD on Bent was around $106 \mathrm{mg} \cdot \mathrm{g}^{-1}$ with $0.3 \mathrm{wt} \%$ HPAD and $1 \mathrm{wt} \%$ Bent in saltwater. Increasing temperature stimulated the desorption of HPAD from Bent in saltwater through increased molecular thermal motion and enhanced thermal degradation of adsorptive groups. At temperatures below $135^{\circ} \mathrm{C}$, the former factor dominated the desorption of HPAD, whereas the latter became more influential at higher temperatures. At higher temperatures, the positively charged quaternary ammonium groups were better adsorptive groups because the amide groups were more prone to decomposition. As the desorption mechanism of $\mathrm{HPAD}$ at $115-175^{\circ} \mathrm{C}$ has been clarified, the next step of our research is to study the conformation of polyampholyte on Bent and enhance the adsorption temperature to over $200^{\circ} \mathrm{C}$. Researchers dealing with the development of polyampholyte-based additives resistant to high temperatures $\left(>150^{\circ} \mathrm{C}\right)$ for drilling ultra-deep wells may benefit from this study.

Acknowledgement: This research was funded by the National Natural Science Foundation of China (41702391) and the Young Scholars Development Fund of SWPU (201599010037).

Author contributions: Design of the study: L.L.; The execution of the study: L.L. X.L.C.S. and Y.M.; The data analysis L.L. and X.L.; The writing of the manuscript: L.L. and X.L.

Conflicts of interest: The authors declare no conflict of interest.

\section{References}

1. Lin L., Luo P., Amphoteric hydrolyzed poly(acrylamide/dimethyl diallyl ammonium chloride) as a filtration reducer under high temperatures and high salinities. J. Appl. Polym. Sci., 2015, 132, 41581-41591.

2. Chu Q., Lin L., Synthesis and properties of an improved agent with restricted viscosity and shearing strength in water-based drilling fluid. J. Petrol. Sci. Eng., 2019, 173, 1254-1263.
3. Lin L., Luo P., Effect of polyampholyte-bentonite interactions on the properties of saltwater mud. Appl. Clay Sci., 2018, 163, 10-19.

4. Chu Q., Lin L., Effect of molecular flexibility on the rheological and filtration properties of synthetic polymers used as fluid loss additives in water-based drilling fluid. RSC Adv., 2019, 9, 8608-8619.

5. Fechner M., Koetz J., Polyampholyte/surfactant complexes at the water-air interface: a surface tension study. Langmuir, 2013, 29, 7600-7606.

6. Mahltig B., Gohy J., Jérôme R., Stamm M., Diblock polyampholytes at the silicon-water interface: Adsorption as a function of block ratio and molecular weight. J. Polym. Sci. Pol. Phys., 2015, 39, 709-718.

7. Tekin N., Dinçer A., Demirbaş Ö., Alkan M., Adsorption of cationic polyacrylamide (C-PAM) on expanded perlite. Appl. Clay Sci., 2010, 50, 125-129.

8. Lin L., Luo Y., Li X., Synthesis of diblock polyampholyte PAMPSb-PMAPTAC and its adsorption on bentonite. Polymers-Basel, 2019, 11, 41-53.

9. Yan X., Zhang X., Interactive effects of clay and polyacrylamide properties on flocculation of pure and subsoil clays. Soil Res., 2014, 52, 727-737.

10. Wang W., Zheng B., Deng Z., Feng Z., Fu L. Kinetics and equilibriums for adsorption of poly(vinyl alcohol) from aqueous solution onto natural bentonite. Chem. Eng. J., 2013, 214, 343-354.

11. Daifullah A.A.M., Girgis B.S., Gad H.M.H., A study of the factors affecting the removal of humic acid by activated carbon prepared from biomass material. Colloid. Surface. A, 2004, 235, 1-10.

12. Lian L., Guo L., Guo C., Adsorption of Congo red from aqueous solutions onto Ca-bentonite. J. Hazard. Mater., 2009, 161, 126-131.

13. Huang J., Liu Y., Jin Q., Wang X., Yang J., Adsorption studies of a water soluble dye, Reactive Red MF-3B, using sonicationsurfactant-modified attapulgite clay. J. Hazard. Mater., 2007, 143, 541-548.

14. Jiang Z., Su Z., Li L., Zhang K., Huang G., Antiaging Mechanism for Partly Crosslinked Polyacrylamide in Saline Solution under High-Temperature and High-Salinity Conditions. J. Macromol. Sci. B, 2012, 52, 113-126.

15. Guan S., Fan H., Duan J., Song C., Examination the Concentration of HPAM - the Starch-Cadmium lodine Method. Journal of Daqing Petroleum Institute, 2007, 31, 110-112.

16. Cai T., Yang Z., Li H., Yang H., Li A., Cheng R., Effect of hydrolysis degree of hydrolyzed polyacrylamide grafted carboxymethyl cellulose on dye removal efficiency. Cellulose, 2013, 20, 2605-2614.

17. Kheradmand H., François J., Plazanet V., Hydrolysis of polyacrylamide and acrylic acid-acrylamide copolymers at neutral $\mathrm{pH}$ and high temperature. Polymer, 1988, 29, 860-870.

18. Smets G., Hesbain A.M., Hydrolysis of polyacrylamide and acrylic acid-acrylamide copolymers. J. Polym. Sci., 1959, 40, 217-226. 